\title{
Oncogenes, Growth Factor
}

National Cancer Institute

\section{Source}

National Cancer Institute. Oncogenes, Growth Factor. NCI Thesaurus. Code C18341.

Mutated forms of growth factor genes which encode a diverse group of extracellular regulatory growth factor signaling molecules. These protein products play roles in the division, growth, proliferation, survival and differentiation of cells. Under normal cellular conditions, the activity of these proteins is tightly controlled. Activation of growth factor proto-oncogenes via mutation results in growth factor oncogenes. These aberrant genes express protein products that disrupt normal cell function. 